host cells to introduce a double-strand break. References: K Miyazono, Y Furuta, M Watanabe-Matsui, T Miyakawa, T Ito, I Kobayashi, M Tanokura. Nat Commun. 2014 5:3178.

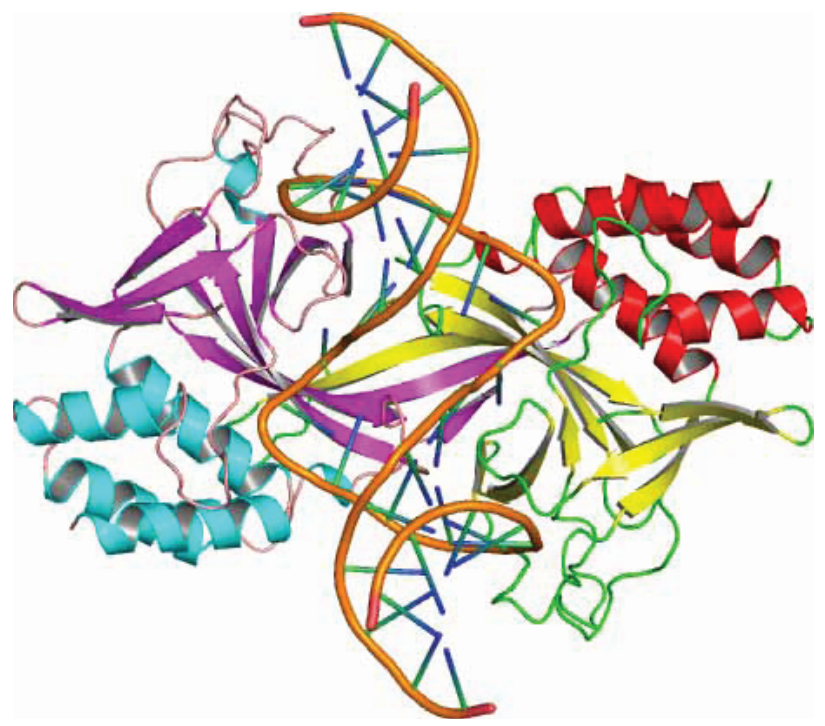

Figure 1. Crystal structure of the R.PabI-DNA complex.

Keywords: restriction enzyme, DNA glycosylase

\section{MS5-P29 Structure-function relationship of zinc-dependent \\ 3'nucleotidases/endonucleases}

\author{
Jan Dohnalek ${ }^{1}$, Tomáš Koval ${ }^{1,2}$, Mária Trundová ${ }^{1}$, Jan Stránský ${ }^{1}$, \\ Karla Fejfarová ${ }^{1,2}$, Petr Kolenko ${ }^{1,2}$, Jarmila Dušková ${ }^{1}$, Tereza \\ Skálová ${ }^{1}$, Jindřich Hašek ${ }^{1}$
}

1. Institute of Biotechnology AS CR, Vídeňská 1083, 14220 Prague, Czech Republic

2. Institute of Macromolecular Chemistry AS CR, Heyrovského nám. 2, 16206 Prague, Czech Republic

email: dohnalek007@gmail.com

Our earlier structure-function studies of tomato bifunctional nuclease 1 (TBN1) showed several key characteristics of this nuclease capable of cleaving single strand and double strand DNA, RNA and structured RNA and also capable of 3'nucleotidase activity (Koval et al., 2013). TBN1 belongs to a wider group of zinc dependent nucleases from plants, bacteria and eukaryotic pathogens. Their natural role is in scavenging of nutrients, specific apoptotic processes and senescence in plants or in pathogen - host interactions. Our x-ray structure of the TBN1 enzyme provided a detailed view of the active site and rather unusual and repeated occlusion of the active site in crystal structures by a surface loop of a neighbouring enzyme molecule (Koval et al., 2013). Other structural studies were performed by other groups for Penicillium citrinum DNase P1 (Romier et al., 1998) and for an Arabidopsis thaliana endonuclease ( $\mathrm{Yu}$ et al., 2014). The known structures and functional data for all these nucleases prove presence of more or less identical active centre (trinuclear zinc cluster) but show different substrate specificity and modulation of the active site around the catalytic centre, together with varied $\mathrm{pH}$ optima and substrate preference/specificity. Attempts were made to describe in detail the catalytic mechanism of this enzyme but our recent data show that our understanding of substrate binding, cleavage and product release is limited and further experiments and analysis are necessary. Here we undertake the task of mapping known structural data onto other known sequences of nucleases of the same type. The main questions include the variability of the role of glycosylation, formation of stabilizing disulfide bridges, presence and nature of the first and second base-binding site, enzyme maturation and especially substrate specificity and inhibition. Our project is focused on structural characterization of Legionella pneumophila 3'nucleotidase and its inhibition and related enzymes from trypanosomatids. Recently, we have successfully cloned and expressed this enzyme which under normal conditions is toxic for the producing bacterium.

The project is supported by BIOCEV CZ.1.05/1.1.00/02.0109 ERDF, and MSMT projects EE2.3.30.0029 and LG14009.

1. Koval, T., Lipovova, P., Podzimek, T., et al. (2013) Acta Cryst. D69, 213-226.

2. Romier, C., Dominguez, R., Lahm, et al. (1998) Proteins, 32, 414-424.

3. Yu T-F., Maestre-Reyna M, Ko, C-Y., et al. (2014) PLOS ONE 9(8): e105821.

Keywords: 3'nucleotidase, endonuclease, zinc cluster, substrate specificity, human pathogens, Legionella 